\title{
Die Freigabe des autonomen Fahrens
}

\author{
Walther Wachenfeld, Hermann Winner
}

\section{Inhaltsverzeichnis}

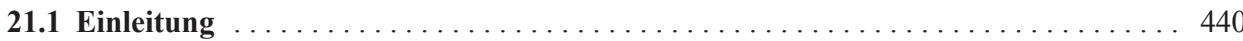

21.2 Aktuelle Testkonzepte in der Automobilindustrie $\ldots \ldots \ldots \ldots \ldots \ldots \ldots \ldots \ldots$

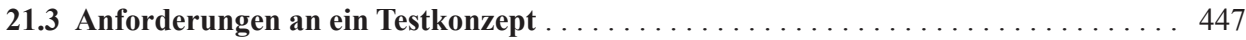

21.3.1 Effektivitätskriterien . . . . . . . . . . . . . . . . . . . . . 447

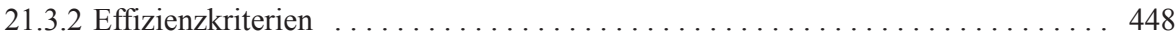

21.4 Besonderheiten des autonomen Fahrens $\ldots \ldots \ldots \ldots \ldots \ldots \ldots \ldots \ldots \ldots \ldots \ldots$

21.4.1 Vergleich zwischen aktueller Automatisierung und Vollautomatisierung der Straßenfahrzeuge . . . . . . . . . . . . . . . . . . 449

21.4.2 Vergleich der Bedingungen in der Luftfahrt, im Straßenverkehr und im Eisenbahnverkehr . . . . . . . . . . . . . . 451

21.5 Freigabeherausforderung für das vollautomatisierte Fahren (Freigabefalle) . . . . 451

21.5.1 Aussagekraft des aktuellen Testkonzepts für das autonome Fahren . . . . . . . . . 453

21.5.2 Millionen Kilometer auf öffentlichen Straßen bis zur Freigabe des vollautomatischen Fahrens . . . . . . . . . . . . . . . . . . . . . . . . . . 454

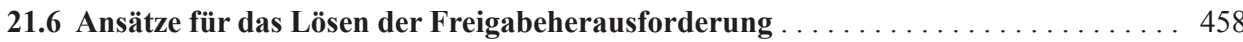

21.6.1 Wiederverwenden freigegebener Funktionen . . . . . . . . . . . . . . . 458

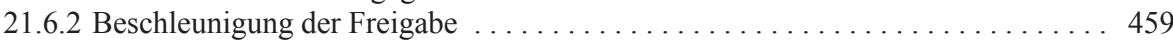

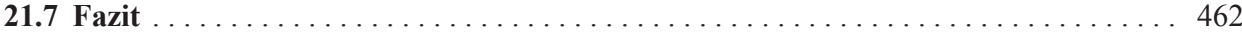

Literatur ....................................... 463

W. Wachenfeld $(\square)$

Technische Universität Darmstadt, Fachgebiet Fahrzeugtechnik - FZD, Deutschland wachenfeld@fzd.tu-darmstadt.de

H. Winner

Technische Universität Darmstadt, Fachgebiet Fahrzeugtechnik - FZD, Deutschland winner@fzd.tu-darmstadt.de 


\subsection{Einleitung}

Die Funktionen des autonomen Fahrens könnten in Zukunft den gesamten Straßenverkehr grundlegend verändern; dafür müssen diese in einer großen Stückzahl und somit als Serienprodukte zum Einsatz kommen. Im Allgemeinen ist für den Übergang eines technischen Systems von der Entwicklungsphase in die Serienproduktion die Freigabe dieses Systems notwendig [1]. Nach den Grundsätzen des Projektmanagements erfolgt die Freigabe erst dann, wenn die zuvor definierten Anforderungen von diesem technischen System erfüllt werden. Diese Anforderungen haben unterschiedlichste Ursprünge, wie beispielsweise Kunden, Normen oder Gesetze. Verschiedene Bereiche werden durch die Anforderungen adressiert: Darunter fallen nicht zuletzt aus Gründen der Typgenehmigung ${ }^{1}$ als auch der Produkthaftung ${ }^{2}$ Anforderungen an die Sicherheit des technischen Systems.

Die Sicherheit des Menschen im öffentlichen Straßenverkehr ist eine der viel zitierten Motivationen für die Fahrzeugautomatisierung, denn die weit überwiegende Zahl der aktuellen Unfälle wird durch den menschlichen Fahrer verursacht. Aus dieser Motivation folgt die Anforderung, dass die Substitution des Menschen die Sicherheit im öffentlichen Straßenverkehr nicht mindert. Dies soll sowohl für den Insassen als auch für das gesamte Verkehrssystem gültig sein, in dem sich das autonome Fahrzeug bewegt. Was diese Anforderung bedeutet und ob sie tatsächlich bei Einführung gestellt wird, steht im Fokus der folgenden Diskussion.

Überlegungen von Gasser et al. [4] liefern hierzu einen Einstieg: Der Bericht der Bundesanstalt für Straßenwesen betrachtet die Entwicklung der Unfallzahlen mit Einführung der Fahrzeugautomatisierung. Ausgehend von der Gesamtzahl der Unfälle bei konventioneller Fahrzeugführung (s. Abb. 21.1 blaues und grünes Feld) wird angenommen, dass Unfälle (grünes Feld) durch die Fahrzeugautomatisierung vermieden werden. Zusätzlich könnten jedoch neue Unfälle aufgrund von Automatisierungsrisiken entstehen (gelbes Feld).

Bei dieser Darstellung wird nicht nach der Schwere des Unfalls unterschieden; jedoch ist die Schwere eines Unfalls bei Betrachtung der Auswirkungen auf die Sicherheit ebenfalls von Relevanz. Sicherheit wird allgemein als Abwesenheit von unangemessenen Risiken beschrieben. Dieses Risiko ist definiert als Produkt aus Wahrscheinlichkeit für einen Unfall und Schadenschwere infolge des Unfalls.

Abb. 21.2 illustriert dieses theoretische Risikovermeidungspotenzial abhängig von der Schwere eines Unfalls in qualitativer Weise. Sie folgt dabei den Erkenntnissen von

1 Im Sinne der Richtlinie 2007/46/EG [2] bezeichnet der Ausdruck „..., Typgenehmigung“ das Verfahren, nach dem ein Mitgliedstaat bescheinigt, dass ein Typ eines Fahrzeugs (...) den einschlägigen Verwaltungsvorschriften und technischen Anforderungen entspricht".

2 Reuter schreibt: „Die (deliktische) Produkthaftung dient dem Schutz von jedermann (Produktnutzer sowie unbeteiligte Dritte) vor unsicheren Produkten. Die Produkthaftung regelt den Ersatz von Folgeschäden, die als Folge der Verletzung von Gesundheit oder Eigentum durch Produktfehler verursacht wurden.“ [3] 


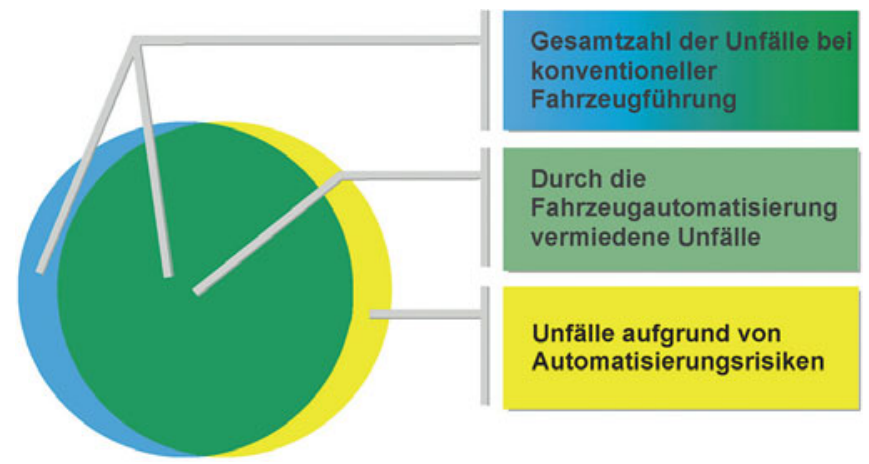

Abb. 21.1 Theoretisches Unfallvermeidungspotenzial bei Fahrzeugautomatisierung [4]

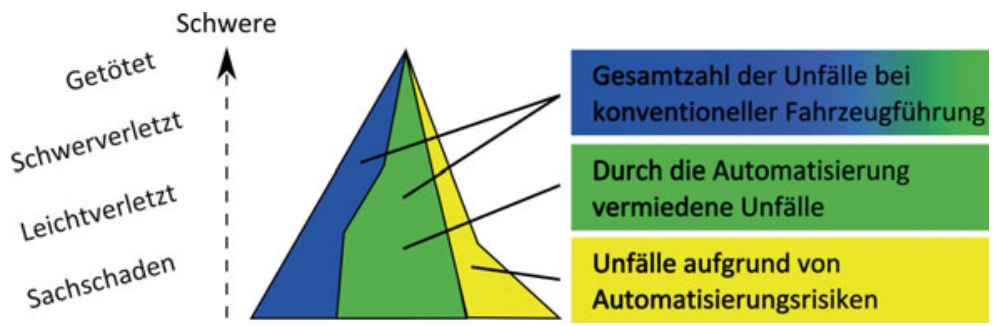

Abb. 21.2 Theoretisches Unfallvermeidungspotenzial bei Fahrzeugautomatisierung unter Berücksichtigung der Unfallschwere (nach [4])

Heinrich [5] und Hydén [6], dass Unfälle mit abnehmender Schwere in größerer Zahl auftreten. Die Skala der zugehörigen Schwere des Unfalls ist ordinal, d.h., eine Ordnung zwischen den verschiedenen Schweregraden ist eindeutig vorhanden: Ein Getöteter wiegt beispielsweise schwerer als ein Schwerverletzter. Das Verhältnis zwischen den Stufen ist jedoch in der Wissenschaft strittig. Zwar werden Schweregrade über Kosten verglichen, dies ist jedoch umstritten und wird in dieser Arbeit nicht weiter diskutiert.

Aus der Betrachtung von Schwere und Anzahl folgt, dass zwar Risiken behoben werden (s. Abb. 21.2 grüner Bereich), aber auch weiterhin Risiken existieren (s. Abb. 21.2 blauer Bereich), die nicht durch die Fahrzeugautomatisierung adressiert werden. Zusätzlich entstehen neue Risiken durch die Substitution des Menschen und die automatisierte Ausführung der Fahrzeugführung. Der Mensch steht nicht mehr als Rückfallebene im Fall eines Fehlers oder einer Unzulänglichkeit zur Verfügung. Abb. 21.2 stellt dieses zusätzliche Risiko durch den gelben Bereich dar. Hierbei ist ungewiss, ob das Beheben von Risiken und das Entstehen zusätzlicher Risiken über die Schwere gleichmäßig erfolgt. Möglich ist eine stärkere Abnahme von schweren Unfällen, aber eine Zunahme von leichteren Unfällen. Abb. 21.2 zeigt diesen Gedanken mithilfe der Verformung des angenommenen Dreiecks. 
Für die Freigabe des vollautomatisierten Fahrens bedeutet dies, dass nicht nur eine Reduktion der Unfallzahlen nachzuweisen ist, sondern ein akzeptiertes Verhältnis $V_{a k z}$ zwischen vermiedenen $R_{\text {ver }}$ und zusätzlich hervorgerufenen Risiken $R_{z u s}$.

$$
V_{a k z}=\frac{R_{z u s}}{R_{v e r}}
$$

Entgegen vieler Aussagen ist bisher noch nicht nachgewiesen, dass ein Verhältnis kleiner eins tatsächlich für die Freigabe notwendig ist. Werden autonome Fahrzeuge tatsächlich die Verkehrssicherheit erhöhen? Wäre das Verhältnis größer eins, würde durch das System die Verkehrssicherheit reduziert. Heute existieren Beispiele, bei denen ein entsprechender Zusatznutzen Akzeptanz für zusätzliche Risiken schafft: Das Erleben von Freiheit, Fahrspaß usw. wiegt beispielsweise für viele Motorradfahrer das erhebliche Zusatzrisiko gegenüber anderen Fortbewegungsmitteln auf, wobei der Akzeptanz des Motorradfahrens die Verteilung von Nutzen und Risiko zugutekommt. Der Zusatznutzen sowie das Zusatzrisiko betreffen überwiegend die Person auf einem Motorrad. Das von einem Motorrad ausgehende Risiko für andere Verkehrsteilnehmer liegt zwischen den Risiken, die von Fahrrad und Pkw ausgehen, sodass Motorradfahren auch ohne Zusatznutzen für andere Verkehrsteilnehmer akzeptabel ist.

In diesem Beitrag wird kein konkreter Wert des akzeptablen Verhältnisses für autonomes Fahren ermittelt, denn dieser Wert ist das Ergebnis einer vielschichtigen Diskussion der Beteiligten, die vom autonomen Fahren betroffen wären. Dieser Wert variiert, bedingt durch unterschiedliche Faktoren wie etwa gesellschaftliche, politische und ökonomische Unterschiede. Ein anschauliches Beispiel hierfür ist die Akzeptanz des Einsatzes von Atomenergie in Deutschland, den USA oder Japan über die letzten Jahre hinweg: Zum einen unterscheidet sich das akzeptierte Verhältnis zwischen den Ländern grundlegend und zum anderen verändert es sich über die Zeit, sodass beispielsweise in Deutschland 2012 der Ausstieg aus der Atomenergie beschlossen wurde.

Das Zentrum dieses Beitrags bildet die Bewertung des autonomen Fahrens, also die Frage nach Methoden, die eine Freigabe ermöglichen sollen. Auch wenn eine Vielzahl von Arbeiten die Potenziale des autonomen Fahrens theoretisch beschreiben, ist den Autoren keine Untersuchung bekannt, die diese Bewertung erbracht hat. Um zu zeigen, warum dies so ist, werden zunächst die aktuellen Freigabekonzepte in der Automobilindustrie beschrieben und anschließend dargestellt, welche Anforderungen an Testkonzepte bestehen. Im dritten Abschnitt werden die Besonderheiten des autonomen Fahrens im Verhältnis zu aktuellen Systemen beschrieben. Basierend darauf wird im vierten Abschnitt die besondere Herausforderung für eine Freigabe des autonomen Fahrens hergeleitet. Die Ansätze, die diese Herausforderung adressieren, werden diskutiert, um im letzten Abschnitt ein Fazit zur Freigabe von autonomen Fahrzeugen zu ziehen. 


\subsection{Aktuelle Testkonzepte in der Automobilindustrie}

Freigabekonzepte, die aktuell in der Automobilindustrie eingesetzt werden, erzielen Freigaben für vier verschiedene Automatisierungsstufen. Um den Unterschied für die Freigabe dieser Systeme im Vergleich zum autonomen Fahren herzuleiten, werden diese vier Systeme kurz erläutert:

Das erste System in Serie ist das Driver-Only-Fahrzeug ohne Automatisierung der Fahraufgabe. Bei diesen Systemen wird auf der einen Seite gezeigt, dass eingesetzte Komponenten maximale Ausfallwahrscheinlichkeiten (Failure Rate) nicht überschreiten und auf der anderen Seite der Fahrer in der Lage ist, das Fahrzeug sicher im Straßenverkehr zu bewegen (Controllability). Dabei wird auf die Fähigkeiten des Fahrzeugführers vertraut, denn die Ergebnisse von ausgeführten Tests mit Testfahrern werden auf zukünftige Nutzer im späteren Einsatzbereich übertragen. Dies hat sich während der letzten Jahrzehnte als erfolgreich erwiesen, um Sicherheit nachzuweisen. Trotz steigender Fahrtkilometer im Straßenverkehr bleibt die Anzahl an Unfällen konstant bzw. ist die Zahl tödlich Verunglückter sogar rückläufig.

Die zweite Stufe der in Serie vorhandenen Automatisierung ist das assistierende System: Für Systeme wie Adaptive Cruise Control (ACC) oder Lane Keeping Assist (LKA) ist zusätzlich zum bestehenden Umfang die Funktion dieser Systeme entsprechend dem ADAS Code of Practice [7] abzusichern. Dabei ist für Systeme, die die primäre Fahraufgabe aktiv unterstützen, den Komfort erhöhen und die Belastung reduzieren, vor allem die Kontrollierbarkeit und die Möglichkeit zur Übernahme zu gewährleisten. Der Code of Practice [7] geht bei diesen ADAS davon aus, dass der Fahrer die Verantwortung für das Verhalten des Fahrzeugs behält. Für diese Systeme gilt erneut, dass auf die Fähigkeiten des Fahrzeugführers vertraut wird, sodass die Ergebnisse von ausgeführten Tests mit Testfahrern auf den späteren Einsatzbereich mit zukünftigen Nutzern übertragen werden.

Ebenfalls freigegeben für den Einsatz in Serienfahrzeugen wurden erste teilautomatisierte Systeme: ACC in Verbindung mit LKA übernimmt abhängig von der Geschwindigkeit Quer- und Längsführung für den Fahrer. Gemäß Definition obliegt die Verantwortung des Fahrzeugverhaltens auch für die dritte Kategorie von Systemen dem Fahrer. Dementsprechend stehen auch für diese Freigabe die Möglichkeit zur Übernahme und die Kontrollierbarkeit durch den Fahrer im Fokus; somit gilt der gleiche Grundsatz wie für das assistierende System, der auf die Fähigkeiten des Fahrzeugführers zur Korrektur von ungewünschtem Automatisierungsverhalten vertraut. Diese Stufe der Automatisierung besitzt die besondere Herausforderung für die Freigabe, die aus dem Konflikt zwischen Entlastung des Fahrers und notwendigem Situationsbewusstsein des Überwachers von Quer- sowie Längsführung folgt. Jedoch liegt auch hierbei letztlich die Verantwortung beim Fahrer.

Von besonderem Interesse für die Freigabe sind Notreaktionssysteme, die automatisiert in die Fahrzeugregelung und somit in die Fahrdynamik eingreifen. Ziel dieser vierten Kategorie von Systemen ist es, dem Kontrollverlust des Fahrers über die Situation entgegenzuwirken. Beispielsweise sind Electronic Stability Control (ESC) und Emergency Brake Assist (EBA) Teile mechatronischer Bremssysteme und bauen ohne Aktion des 
Abb. 21.3 Prinzipdarstellung einer ReceiverOperating-Characteristic-Kurve (nach [8])

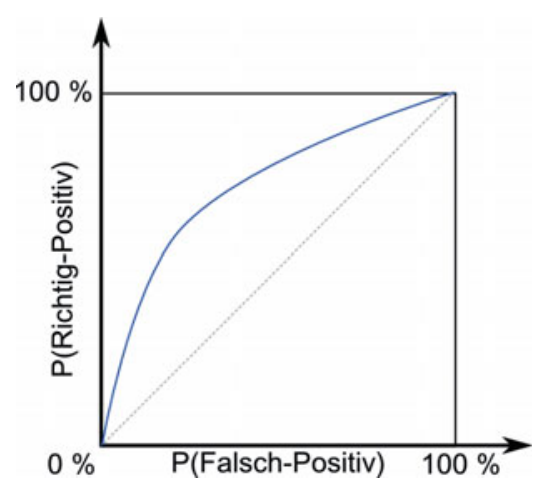

Fahrers zusätzliche Bremskraft auf bzw. ab und greifen somit aktiv in die Fahrdynamik ein. Dies geschieht während des Kontrollverlusts durch den Fahrer, wenn das Fahrzeug in Verbindung mit dem Fahrer einem erhöhten Risiko ausgesetzt ist. ESC wird so ausgelegt, dass eingegriffen wird, wenn das Fahrzeug vom Fahrer in der aktuellen Situation offensichtlich nicht mehr beherrscht wird (z. B. bei sehr starkem Über- oder Untersteuern). Der EBA wird hingegen dann aktiv, wenn Reaktionszeit und Bremsweg bis zu einem Auffahrunfall für den Menschen nicht mehr ausreichen, diesen Unfall zu verhindern. Ziel für die Absicherung ist es zu zeigen, dass Notreaktionssysteme möglichst nur dann aktiv werden (RichtigPositiv-Rate), wenn der Kontrollverlust offensichtlich wird und somit ein stark erhöhtes Risiko herrscht. Dafür ist zu zeigen, dass die Falsch-Positiv-Rate möglichst klein wird und/ oder die Auswirkungen vom Fahrer kontrolliert werden können; Falsch-Positiv- und Falsch-Negativ-Raten der EBA hängen vor allem von der Objekterkennung ab. In Abb. 21.3 ist eine Receiver-Operating-Characteristic-Kurve (ROC-Kurve) dargestellt, die diesen Zusammenhang für die Objekterkennung beschreibt.

Da es sich bei diesen Notreaktionssystemen um Systeme ohne Funktionsversprechen handelt, kann eine Erhöhung der Sicherheit durch Reduktion des Nutzens und eine kleinere Falsch-Positiv-Rate erzielt werden. Zusätzlich ermöglichen diese Systeme auch ein Übersteuern. ESC und EBA greifen über das gezielte Abbremsen von Rädern nur in ein Stellsystem ein und können mit unterschiedlichen Strategien durch Lenken und/oder Beschleunigen übersteuert werden.

Wie gezeigt wurde, steht bei der Entwicklung der vier Systemstufen die Kontrollierbarkeit durch den Fahrer im Mittelpunkt: Entweder geht es darum, dem Fahrer die Kontrollierbarkeit zu ermöglichen oder sie für ihn wiederherzustellen (Design for Controllability). Der Fahrer als Rückfallebene ist somit Grundlage für die Absicherung aktueller Fahrzeuge.

Die Entwicklung und der Nachweis dieser Kontrollierbarkeit für den Fahrer erfolgt meist entsprechend dem Vorgehensmodell in Abb. 21.4. Dieses Vorgehen nach dem V-Modell unterscheidet zwischen dem linken absteigenden Ast der Entwicklung und Auslegung und dem rechten aufsteigenden Ast der Verifikation und Validierung als Mittel zur Qualitätssicherung. Für die Qualitätssicherung wird dabei einem Testkonzept gefolgt. 


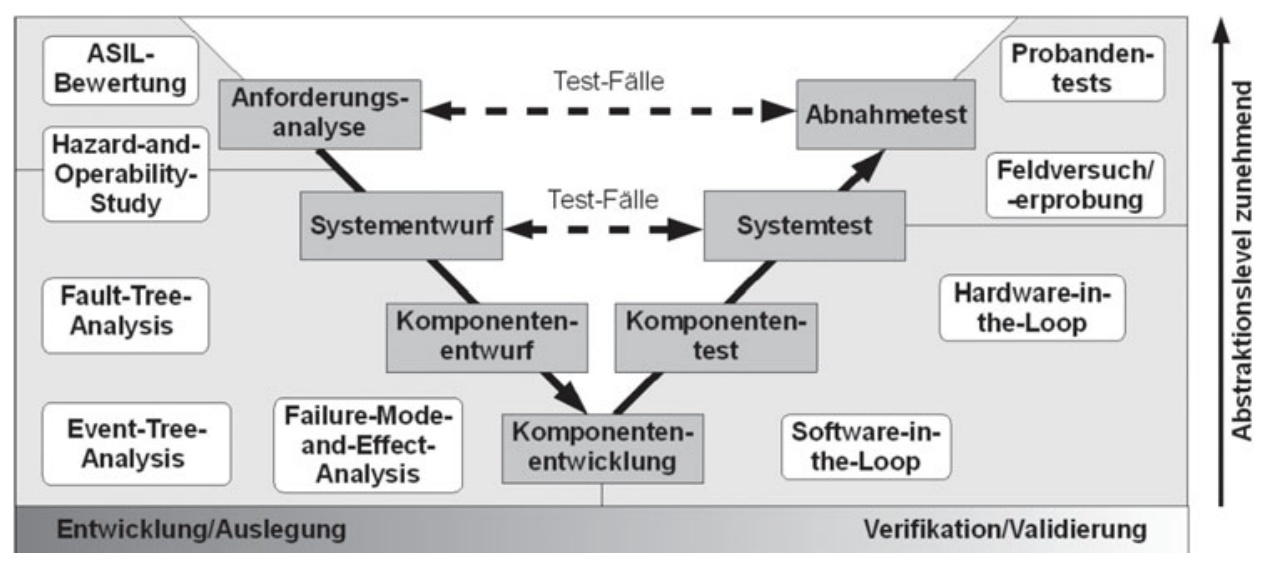

Abb. 21.4 Einordnung von Sicherheitsbewertungsmethoden im Entwicklungsprozess (nach [9])

Ein Testkonzept umfasst, wie von Schuldt et al. [10] in Abb. 21.5 dargestellt, die Analyse des Testobjekts (object under test - OUT), die Testfallgenerierung, die Testdurchführung sowie die Testauswertung.

Dabei sollten die Analyse des Testobjekts sowie die Testfallgenerierung bereits während der Entwicklungs-/Auslegungsphase stattfinden, sodass für die Verifikation und Validierung die durchzuführenden Testfälle bereits definiert sind (s. Abb. 21.4 V-Modell). Nach Horstmann [11] und Weitzel [9] wird aktuell für die Ermittlung von Testfällen zwischen drei Methoden unterschieden: Eine Methode ist die lastenheftbasierte Testspezifikation, bei welcher Testfälle basierend auf Systemspezifikationen definiert werden, die beispielsweise in Lastenheften festgehalten wurden. Die zweite Methode stellt die risikobasierte Testspezifikation dar, bei der Risikobetrachtungen für die Testfallermittlung eingesetzt werden. Als dritte Methode wird die schnittstellenbasierte Testspezifikation unterschieden, bei der die Testfälle so gewählt werden, dass sie die Wertebereiche der Schnittstellen entsprechend abdecken. Für alle Methoden ist das System Fahrer - Fahrzeug die Grundlage der Testfallermittlung.

Abb. 21.5 Vorgehensweise des Testkonzepts (nach [10])

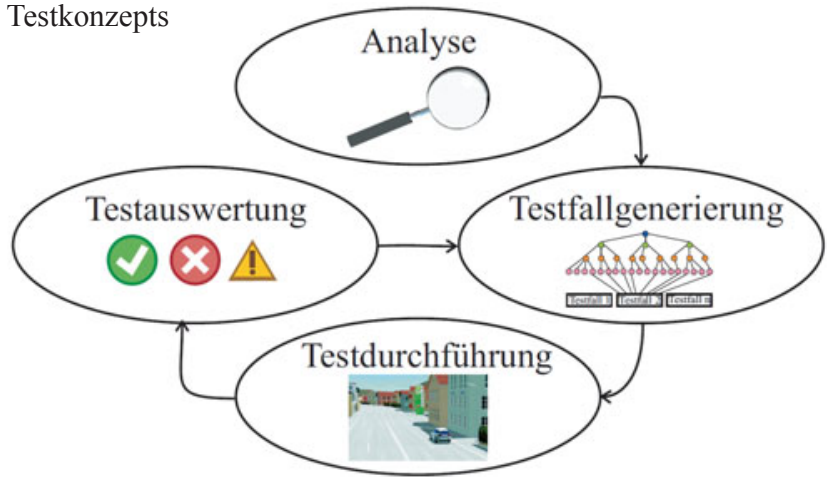


Um mit der Qualitätssicherung möglichst frühzeitig zu beginnen, werden bereits, bevor die ersten Testfahrzeuge erprobungstauglich sind, Tests in virtuellen Testumgebungen durchgeführt. Die Testdurchführung mittels Modell- und Software-in-the-Loop prüft Funktionen basierend auf Simulationsmodellen des Fahrzeugs, des Menschen sowie des Umfelds. Dabei werden die zuvor identifizierten Testfälle eingesetzt. Je weiter die Entwicklung voranschreitet, desto mehr reale Komponenten stehen zur Verfügung, die einem Test unterzogen werden: Hierbei kommen Prüfstände, Fahrsimulatoren oder Testgelände zum Einsatz. Die Testdurchführung mittels Hardware-in-the-Loop, Driver-in-the-Loop oder Vehicle-in-the-Loop geben Auskunft über die Qualität der unter Test stehenden Komponenten und Funktionen. Um Aktion und Reaktion des Systems Fahrer - Fahrzeug-Umwelt zu überprüfen (den Loop zu schließen), werden auch für diese Testdurchführungen Simulationsmodelle eingesetzt. Daher werden bis zu diesem Entwicklungszeitpunkt für die Testdurchführung stets Simulationsmodelle benötigt, um das Gesamtfahrzeug zu testen. Simulationsmodelle sind Abbildungen der Realität in Software und haben per se die Eigenschaft, die Komplexität der realen Welt zu vereinfachen.

Diese Tatsache führt dazu, dass aktuell keine sicherheitsrelevante Funktion in einem Serienfahrzeug existiert, die nicht auch mit realen Testfahrzeugen überprüft wurde: Deshalb wird für aktuelle Systeme immer auf den Test mit realen Fahrzeugen, realen Menschen und realer Umwelt zurückgegriffen.

Aus dem notwendigen Einsatz der Realfahrt folgt, dass beispielsweise vor der Freigabe der Mercedes Benz E-Klasse (W212) insgesamt 36 Millionen Testkilometer absolviert wurden [12] $]^{3}$. Nach Fach et al. [13] bedarf allein die Freigabe eines aktuellen Fahrerassistenzsystems bis zu zwei Millionen Testkilometer. Wenn zwischen zwei Aktivierungen der ersten Stufe des EBA bei diesen Testfahrten 50000 bis 100000 Kilometer vergingen, wird diese große Zahl an Testkilometern nachvollziehbar. Dabei ist noch nicht berücksichtigt, dass die kritischere zweite Stufe des EBA während dieser Testkilometer gar nicht auslöste (s. Aussage in Abb. 21.3). Diese Testkilometer im zweistelligen Millionenbereich gehen einher mit beträchtlichen Kosten für Fahrzeugprototypen, Testfahrer, Testdurchführung sowie deren Auswertung. Der Zeitbedarf lässt sich zwar durch Parallelerprobungen mittels mehrerer Fahrzeuge reduzieren, jedoch entstehen hierbei zusätzliche Kosten für die Fahrzeugprototypen.

Dieses Beispiel zeigt, dass bereits für aktuelle Fahrerassistenzsysteme die Absicherung basierend auf Realfahrten im Straßenverkehr eine ökonomische Herausforderung für den OEM (engl. Original Equipment Manufacturer) darstellt. Besonders vor dem Hintergrund der zunehmenden Funktionalitäten und der Varianten- sowie Versionsvielfalt je Fahrzeugmodell wächst diese Herausforderung. Burgdorf [14] leitet beispielsweise eine Zahl von $160 \cdot 2^{70}$ Varianten für den BMW $318 \mathrm{i}$ (E90) mit Komponenten wie beispielsweise Karosserieform, Motor, Getriebe, Abtrieb, Farbe, Klima, Infotainment her.

3 „Hinter [der E-Klasse] liegen umfassende virtuelle Tests mit digitalen Prototypen und insgesamt 36 Millionen Testkilometer ....“ 
Deshalb gibt es bereits heute Bestrebungen, andere Testdurchführungen neben der Realfahrt für die finale Absicherung einzusetzen. Das einzige den Autoren dafür bekannte Beispiel betrifft die Homologation von ESC-Systemen. Nach EU-Verordnung ECE-Regelung 13H [15] besteht die Möglichkeit, diese teilweise in der Simulation durchzuführen:

Wenn ein Fahrzeug gemäß Absatz 4 physikalisch geprüft worden ist, kann die Übereinstimmung anderer Versionen oder Varianten desselben Fahrzeugtyps mittels Rechnersimulationen nachgewiesen werden, die die Prüfbedingungen des Absatzes 4 und die Prüfverfahren des Absatzes 5.9 einhalten.

Wohlgemerkt geht es hier allein um das ESC-System. Als Beispiel wird von Baake et al. [16] die Homologation von ESC-Systemen für Vans von Mercedes-Benz in Zusammenarbeit mit Bosch und IPG CarMaker beschrieben: Mithilfe von sogenannten Master Cars wurde in CarMaker ein Fahrzeugmodell erstellt; mit diesen Master Cars wurden Referenzdaten gesammelt und basierend darauf das Simulationsmodell validiert. Damit wurde die simulationsbasierte Empfehlung zur Freigabe für weitere Fahrzeugvarianten mit unterschiedlicher Beladung ermöglicht. Baake et al. berichtet zusätzlich vom Übertragen dieses Vorgehens auf die Funktion Cross Wind Assist (CWA), die jedoch noch nicht für die Absicherung zur Freigabe eingesetzt wurde.

\subsection{Anforderungen an ein Testkonzept}

Um im Folgenden zu diskutieren, warum die Vollautomatisierung eine besondere Herausforderung für die Freigabe darstellt, werden zunächst die Anforderungen an Testkonzepte für die Freigabe beschrieben. Diese sind unterteilt in Effektivitäts- und Effizienzkriterien.

\subsubsection{Effektivitätskriterien}

\section{Repräsentativ - valide}

Die Anforderung der Repräsentativität hat zwei Aspekte: Auf der einen Seite muss die Testfallgenerierung dafür sorgen, dass die nötige Testabdeckung erreicht wird. Ein Fahrzeug sollte beispielsweise nicht nur bei $20^{\circ} \mathrm{C}$ und Sonnenschein getestet werden, da es im realen Einsatz auch Schnee, Regen und Temperaturen unter $0^{\circ} \mathrm{C}$ ausgesetzt ist. Außerdem sollten Fahrzeuggrenzmuster (Toleranzen in der Produktion) bei der Testfallgenerierung betrachtet werden. Auf der anderen Seite muss die Testdurchführung den minimal notwendigen Grad an Realität aufweisen. Das bedeutet, dass die Vereinfachung in der Darstellung der Realität weder das Verhalten des Testobjekts noch das Verhalten und die Eigenschaften der Umwelt gegenüber dem realen Verhalten beeinflussen darf. 


\section{Variierbar}

Die Testdurchführung muss die Möglichkeit bieten, alle von der Testfallgenerierung definierten Testfälle umzusetzen.

\section{Beobachtbar}

Besonders für die Testauswertung ist es notwendig, Parameter der Testdurchführung zu beobachten. Nur wenn die Situation auch beschrieben werden kann, besteht die Möglichkeit zur Aussage „Test bestanden“ oder „Test nicht bestanden“.

\subsubsection{Effizienzkriterien}

\section{Ökonomisch}

Die Anforderung des ökonomischen Testkonzepts ist zweigeteilt: Zum einen sollte die Testdurchführung möglichst schnell vorbereitet und durchgeführt sein, um nach Möglichkeit die an der Entwicklung beteiligten Personen direkt mit einer Rückmeldung zum Testobjekt zu versorgen. Zum anderen ist darauf zu achten, dass die Testdurchführung möglichst kostengünstig vorbereitet und durchgeführt wird.

\section{Reproduzierbar}

Die Reproduzierbarkeit reduziert gravierend den Aufwand für Regressionstests. Wurde beispielsweise ein Fehler erkannt und das Testobjekt entsprechend modifiziert, ist das Ziel, das Testobjekt im gleichen Szenario erneut einem Test zu unterziehen.

\section{Frühzeitig}

Je früher im Entwicklungsprozess ein Produkt aussagekräftig getestet werden kann, desto weniger Entwicklungsschritte müssen bei einem eventuellen Fehler erneut durchlaufen werden.

\section{Sicher}

Die Testdurchführung sollte das akzeptierte Risiko aller Testbeteiligten nicht übersteigen. Dies ist besonders bei Realfahrten zu beachten, wenn Verkehrsteilnehmer dem Test ohne Kenntnis ausgesetzt werden.

Die beschriebenen Anforderungen werden durch die aktuellen Testkonzepte ausreichend erfüllt, sodass die vier vorgestellten unterschiedlichen Automatisierungsstufen damit freigegeben werden. Die Rückrufe aller OEM, die Millionen Fahrzeuge betreffen, sind jedoch ein Zeichen dafür, dass diese Testkonzepte keineswegs alles absichern. Sind diese Konzepte auch weiterhin geeignet, um neue Systeme wie das autonome Fahren für den öffentlichen Straßenverkehr abzusichern? An den vorgestellten Anforderungen ändert sich nichts. Wie im Folgenden beschrieben wird, ändert sich jedoch das Testobjekt gravierend. 


\subsection{Besonderheiten des autonomen Fahrens}

Im Folgenden wird zunächst der Unterschied zwischen vollautomatisiertem und aktuellem Fahren im Straßenverkehr erläutert. Daran anschließend wird der Unterschied zwischen den Verkehrssystemen Luftfahrt, Eisenbahn und Straßenverkehr kompakt vorgestellt, der dazu führt, dass nur bedingt Erkenntnisse aus diesen Bereichen übertragen werden können.

\subsubsection{Vergleich zwischen aktueller Automatisierung und Vollautomatisierung der Straßenfahrzeuge}

Für die zuvor beschriebene Absicherung der vier in Serie vorhandenen Automatisierungsstufen stand das Fahrzeug und speziell dessen Kontrollierbarkeit durch den Fahrer im Fokus. In der kombinierten Darstellung des Drei-Ebenen-Modells für zielgerichtete Tätigkeiten des Menschen nach Rasmussen [17] und der Drei-Ebenen-Hierarchie der Fahraufgabe nach Donges [18] in Abb. 21.6 entspricht diese Absicherung den grün hinterlegten Elementen. Getestet werden das Fahrzeug und dessen Verhalten in Längs- und Querrichtung; dabei werden nicht das Verhalten oder die Fähigkeiten des zukünftigen Fahrers getestet, sondern ausschließlich die Möglichkeiten für den Testfahrer, mit Lenk- und Beschleunigungseingriffen das Fahrzeug in den Testfällen zu kontrollieren. Deshalb schneidet die grüne Box nur leicht den Bereich, der für den Fahrer steht.

Für die Vollautomatisierung fallen nun die Fähigkeiten des Fahrers weg, er fungiert auch nicht mehr als Rückfallebene. Die Fahraufgabe, also das Navigieren, Bahnführen und Stabilisieren/Regeln, wird vom Fahrroboter übernommen. Das bedeutet, für das autonome Fahren gibt es keinen Test der Kontrollierbarkeit, sondern ausschließlich den Test der Funktionalität eines technischen Systems. Auf der einen Seite erleichtert es den Test, denn die Unsicherheiten und individuellen Unterschiede des Menschen sind nicht mehr durch den Test abzudecken. Auf der anderen Seite entfällt die Möglichkeit, von Testfällen und Testfahrern auf weitere Anwendungsfälle zu schließen. Für das System entfällt der Mensch, der im Allgemeinen fertigkeitsbasiert, regelbasiert sowie wissensbasiert handelt.

Für die Absicherung aktueller Systeme ist eine Sicherheit nachzuweisen, die sich durch Fahrer und das Fahrzeug in Kombination ergibt; für die Freigabe des Fahrzeugs steht dabei aber aktuell ausschließlich das Fahrzeug im Fokus. Zusätzlich angenommen, aber nicht getestet wird die „Zuverlässigkeit“ des Fahrers. Bei der Absicherung des autonomen Systems ergibt sich die Sicherheit nun ausschließlich aus dem technischen System Fahrroboter und Fahrzeug (gelbes Feld der Abb. 21.6), die es nachzuweisen gilt.

Aus Abb. 21.6 wird ersichtlich, dass dafür zum einen die Aufgabenquantität steigt, die es abzusichern gilt: Der Fahrroboter wird für die unterschiedlichsten Einsatzgebiete (s. Use-Cases Kap. 2) wie Navigieren, Bahnführen und Stabilisieren/Regeln benötigt. Diese Aufgabenquantität wird besonders im öffentlichen Raum ohne Zugangsbeschränkung eine Herausforderung. Auf der anderen Seite ändert sich die Aufgabenqualität des technischen Systems. Aktuelle Systeme sind lediglich ausführend bzw. ständig durch den 


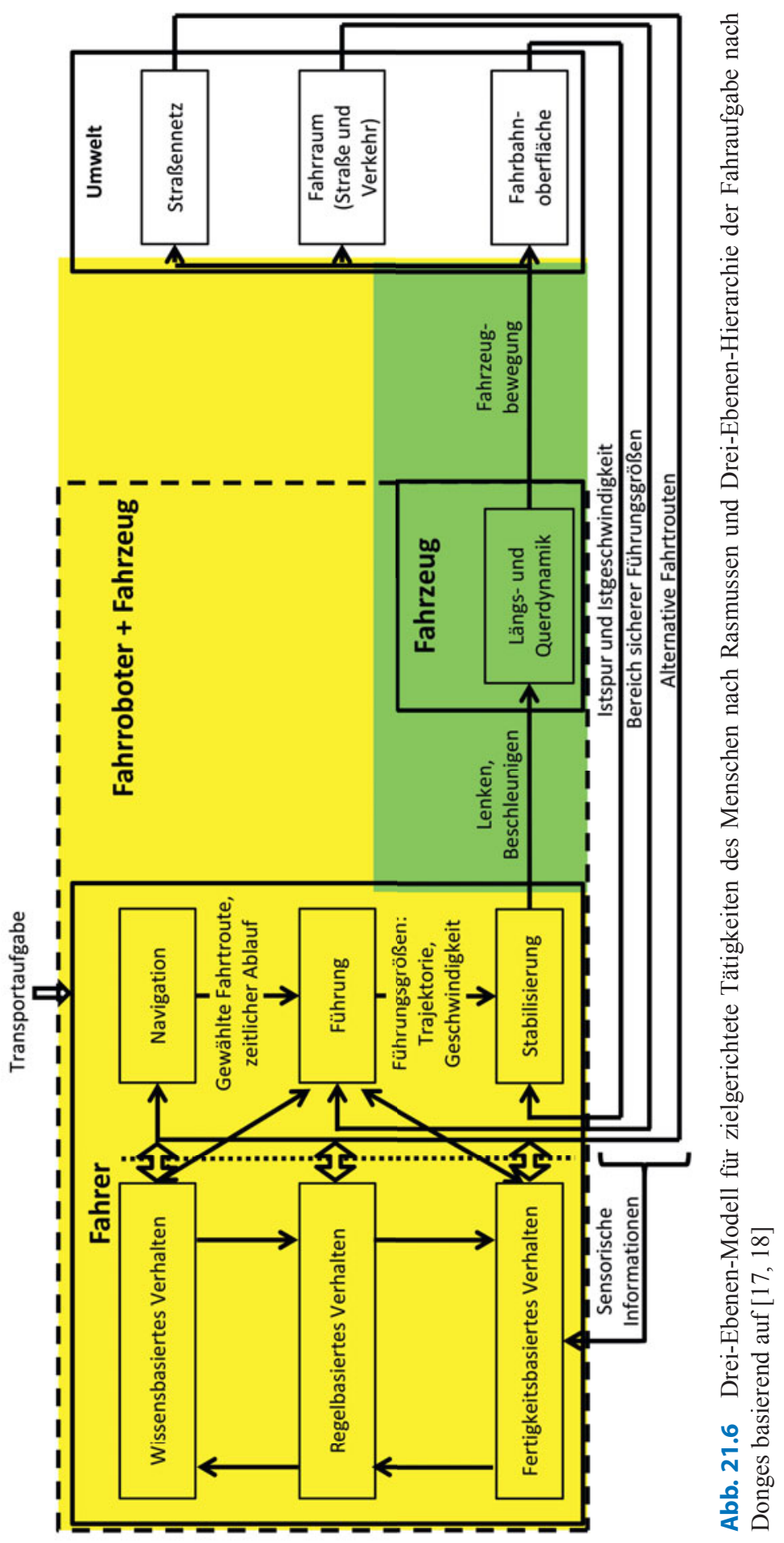


Menschen überwacht, für das autonome System muss die Ausführung einer Aufgabe jedoch den Ansprüchen an die zu Beginn diskutierte Sicherheit genügen.

\subsubsection{Vergleich der Bedingungen in der Luftfahrt, im Straßenverkehr und im Eisenbahnverkehr}

Neben dem Straßenverkehr existieren weitere Verkehrssysteme, bei denen die Automatisierung der Bedienung Einzug gehalten hat. Inwieweit jedoch die Herausforderungen und Lösungen aus diesen Bereichen auf die Fahrzeugautomatisierung übertragbar sind, wird im Folgenden diskutiert.

Die Automatisierung in der (zivilen) Luftfahrt liefert aktuell keine Beispiele der Vollautomatisierung. Auch wenn die Piloten nur äußerst selten tatsächlich der Flugaufgabe nachgehen, sind diese dennoch als Überwacher und Bediener vorhanden. Eine Übersicht über die Unterschiede der Verkehrssysteme findet sich in Tab. 21.1, die Weitzel et al. [9] und Ständer [19] entnommen wurde. Für die Absicherung ist diesbezüglich besonders das Sicherheitskonzept des Verkehrsablaufs von Interesse, denn hier zeigen sich die Unterschiede zwischen Luftfahrt und Straßenverkehr. Die Luftfahrt operiert in einem gesetzlich abgeschlossenen Verkehrsraum, ein Kollisionswarnsystem ist verpflichtend und eine externe Überwachung des Betriebs ist durch die Flugsicherung vorhanden.

Das Verkehrssystem der Eisenbahn bietet Beispiele der Vollautomatisierung: Beispielsweise wird in Nürnberg eine automatisierte U-Bahn betrieben. Jedoch unterscheidet sich auch für dieses Verkehrssystem nach Tab. 21.1 besonders das Sicherheitskonzept des Verkehrsablaufs zwischen Straßenverkehr und Eisenbahn. Für den Bahnverkehr existiert ein gesetzlich abgeschlossener Verkehrsraum, zusätzlich wird mit Logiksystemen und einer externen Überwachung gearbeitet, um die Kollision von zwei Zügen zu vermeiden.

Der Straßenverkehr als Mischbetrieb liefert die Bedingung des abgeschlossenen Verkehrsraumes und der externen Überwachung nicht. Diese Unterschiede zeigen, warum Lösungen für die Freigabe nicht direkt auf das autonome Fahren übertragen werden können.

Mit diesem Vergleich soll nicht ausgeschlossen werden, dass sämtliche Lösungen aus der Luftfahrt und dem Eisenbahnverkehr für den Straßenverkehr uninteressant sind. Sicherlich existieren ähnliche Problemstellungen wie beispielsweise die Zuverlässigkeit von sicherheitsrelevanten Komponenten.

\subsection{Freigabeherausforderung für das vollautomatisierte Fahren (Freigabefalle)}

Wie dargestellt wurde, unterscheidet sich die Funktion des autonomen Fahrens als Testobjekt grundlegend von den aktuellen Straßenfahrzeugen, aber auch von Bewegungsmitteln im Luft- und Eisenbahnverkehr. Deshalb wird nun ermittelt, welche Aussagekraft die vorgestellten aktuellen Testkonzepte übertragen auf das autonome Fahren besitzen 
Tab. 21.1 Vergleich der Bedingungen der Verkehrssysteme (entnommen [9], basierend auf [18])

\begin{tabular}{|c|c|c|c|}
\hline & Luftfahrt & Straßenverkehr & Eisenbahnverkehr \\
\hline Bewegungsoptionen & 3-D (Raum) & 2-D (Fläche) & 1-D (Linie) \\
\hline \multicolumn{4}{|l|}{ Bediener } \\
\hline $\begin{array}{l}\text { verantwortlicher } \\
\text { Fahrzeugführer }\end{array}$ & meist redundant & nicht redundant & nicht redundant \\
\hline $\begin{array}{l}\text { Professionalität } \\
\text { der Fahrzeugführer }\end{array}$ & $\begin{array}{l}\text { fast vollständig } \\
\text { hauptberuflich }\end{array}$ & $\begin{array}{l}\text { geringer Anteil } \\
\text { hauptberuflich }\end{array}$ & $\begin{array}{l}\text { fast vollständig haupt- } \\
\text { beruflich }\end{array}$ \\
\hline \multicolumn{4}{|l|}{ Ausbildung } \\
\hline Theorie & $>750$ Stunden & $>21$ Stunden & $\sim 800$ Stunden \\
\hline Praxis & $>1500$ Stunden & $>9$ Stunden & $\sim 400$ Stunden \\
\hline $\begin{array}{l}\text { Schulung auf } \\
\text { Fahrzeugtyp }\end{array}$ & ja & nein & ja \\
\hline Weiterbildung & erforderlich & nicht erforderlich & erforderlich \\
\hline \multicolumn{4}{|c|}{ Sicherheitskonzepte des Verkehrsablaufs } \\
\hline $\begin{array}{l}\text { Verkehrsraum } \\
\text { abgeschlossen }\end{array}$ & $\begin{array}{l}\text { gesetzlich festge- } \\
\text { legte Begrenzungen }\end{array}$ & in Sonderfällen & $\begin{array}{l}\text { gesetzlich festgelegte } \\
\text { Begrenzungen }\end{array}$ \\
\hline Fahrt auf Sicht & $\begin{array}{l}\text { nein, nur in Sonder- } \\
\text { fällen }\end{array}$ & ja & nein, nur in Sonderfällen \\
\hline $\begin{array}{l}\text { technische Vorrich- } \\
\text { tungen (Beispiele) }\end{array}$ & $\begin{array}{l}\text { Kollisionswarn- } \\
\text { systeme } \\
\text { verpflichtend }\end{array}$ & $\begin{array}{l}\text { Fahrbahnmarkierung, } \\
\text { Lichtsignalanlagen, } \\
\text { Beschilderung }\end{array}$ & $\begin{array}{l}\text { Sicherheitsfahrschal- } \\
\text { tungen, punktförmige } \\
\text { Zugbeeinflussung, } \\
\text { automatische Fahr- und } \\
\text { Bremssteuerung }\end{array}$ \\
\hline $\begin{array}{l}\text { externe } \\
\text { Überwachung }\end{array}$ & ja, Flugsicherung & nein & $\begin{array}{l}\text { ja, Fahrdienstleitung, } \\
\text { Betriebszentrale }\end{array}$ \\
\hline \multicolumn{4}{|c|}{ Technische Rahmenbedingungen } \\
\hline $\begin{array}{l}\text { Dokumentation } \\
\text { Fahrten/ Betriebs- } \\
\text { stunden }\end{array}$ & ja & nein & $\begin{array}{l}\text { Überwachung der Lauf- } \\
\text { leistung, automatische } \\
\text { Fahrtenschreiber }\end{array}$ \\
\hline $\begin{array}{l}\text { Instandhaltung, } \\
\text { Reparatur }\end{array}$ & $\begin{array}{l}\text { nur von zertifizier- } \\
\text { ten Betrieben }\end{array}$ & $\begin{array}{l}\text { Werkstätten, } \\
\text { Selbsthilfe }\end{array}$ & $\begin{array}{l}\text { nur von zertifizierten } \\
\text { Betrieben, dann auch } \\
\text { kleine Werkstätten }\end{array}$ \\
\hline Unfallanalyse & $\begin{array}{l}\text { jeder Unfall/ } \\
\text { schwere Störung, } \\
\text { durch unabhängige } \\
\text { staatliche Stelle }\end{array}$ & $\begin{array}{l}\text { in Einzelfällen, durch } \\
\text { zertifizierte Gutachter }\end{array}$ & $\begin{array}{l}\text { jeder Unfall/ schwere } \\
\text { Störung, durch unabhän- } \\
\text { gige staatliche Stelle }\end{array}$ \\
\hline $\begin{array}{l}\text { Stückzahlen } \\
\text { (in Europa) }\end{array}$ & $10^{3}$ (fallend) & $10^{6}$ (steigend) & $\begin{array}{l}10^{3} \text { (fallend, bei steigen- } \\
\text { der Fahrleistung pro } \\
\text { Triebfahrzeug) }\end{array}$ \\
\hline Modellwechsel & ca. 20 Jahre & ca. 5-7 Jahre & $\begin{array}{l}\text { ca. } 20 \text { Jahre für Trieb- } \\
\text { fahrzeuge }\end{array}$ \\
\hline
\end{tabular}


würden. Des Weiteren wird diskutiert, welche Auswirkungen es hätte, wenn weiterhin am aktuellen Testkonzept festgehalten würde.

\subsubsection{Aussagekraft des aktuellen Testkonzepts für das autonome Fahren}

Es wurde bereits dargelegt, dass ein Testkonzept aus Testfallgenerierung und Testdurchführung besteht. Nun wird diskutiert, wie und ob beides auf das autonome Fahren übertragbar ist.

\section{Testfallgenerierung}

Die drei Verfahren zur Testfallgenerierung wurden in Abschn. 21.2 bereits kurz erläutert; diesen Verfahren liegt die Annahme der Fahrfähigkeit des Fahrers zugrunde. Ob ein beliebiger Fahrer das Testobjekt kontrollieren kann, wird an die gesetzlich vorgeschriebene Fahrerlaubnis geknüpft. Diese Fahrerlaubnis wird nach Straßenverkehrsgesetz ( 2 Abs. 2 StVG) nur dann erteilt, wenn u. a. der Bewerber

- ein Mindestalter erreicht hat,

- zum Führen von Kraftfahrzeugen geeignet ist,

- ausgebildet wurde und

- theoretische wie praktische Tests bestanden hat.

Unter ,geeignet“" wird nach §2 Abs. 4 StVG Folgendes verstanden:

Geeignet zum Führen von Kraftfahrzeugen ist, wer die notwendigen körperlichen und geistigen Anforderungen erfüllt und nicht erheblich oder nicht wiederholt gegen verkehrsrechtliche Vorschriften oder gegen Strafgesetze verstoßen hat.

Aufgrund dieser geforderten Fahrfähigkeit des Fahrers wird die Testfallgenerierung auf Beispielsituationen beschränkt: Angenommen wird, dass, wenn der Testfahrer diese Beispielsituationen bewältigen kann, er bzw. jeder andere Fahrer mit Fahrerlaubnis auch die weiteren nicht getesteten relevanten Situationen im Einsatz bewältigen wird. Dazu gehören Situationen, in denen der Fahrer aktiv fährt, aber auch jene Situationen, in denen der Fahrer überwacht und das System gegebenenfalls übersteuert. Somit bieten die Testfälle in Kombination mit der Fahrerlaubnisprüfung eine Metrik, die eine Aussage über die Sicherheit des Systems Fahrer - Fahrzeug erlaubt. Wie die praktische Fahrerlaubnisprüfung als Bewertungsgrundlage zur Beurteilung der Fahrbefähigung optimiert werden könnte, wird von Bahr [20] diskutiert.

Durch den Wegfall des Fahrzeugführers entfällt die aktuell akzeptierte Metrik, und somit ist die Raffung der Testfälle nicht mehr zulässig. Die Testfallgenerierung für das autonome Fahren muss gerade die Fahrfähigkeiten - eine neue Qualität von Funktionen - abdecken, die der Mensch zuvor mit in das Fahrer-Fahrzeug-System eingebracht hat. Der theoretische und der praktische Test der Fahrerlaubnisprüfung stellen dabei nicht die Schwierigkeit dar. 
Die folgenden Paragrafen - $§ 10$ Mindestalter, $\S 11$ Eignung und $\S 12$ Sehvermögen der Fahrerlaubnis-Verordnung $(\mathrm{FeV})$ - stellen jedoch die Herausforderung dar. Diese Paragrafen stehen damit implizit für umfassende Anforderungen an die Eigenschaften des Menschen, die Fahraufgaben auszuführen. Der Mensch, der diese Anforderungen erfüllt, hat

- als Verkehrsteilnehmer Hunderttausende von Kilometern erlebt,

- als Teil der Gesellschaft deren Sozialverhalten erfahren,

- kognitive Fähigkeiten erlernt,

- sensomotorische Fähigkeiten trainiert usw.

Aktuell ist den Autoren keine Methode bekannt, diese Funktionen für ein technisches System valide zu testen. Somit fallen die akzeptierte Metrik sowie die Raffung der Testfälle weg, wenn der Mensch aus der Verantwortung für die Fahraufgabenausführung genommen wird. Die aktuellen Testfälle besitzen für die Freigabe des autonomen Fahrens keine Aussagekraft, sodass die Testfallgenerierung an das neue System anzupassen ist.

\section{Testdurchführung}

Wie bereits dargelegt, werden für die Testdurchführung unterschiedliche Methoden von Hardware-in-the-Loop (HiL) über Software-in-the-Loop (SiL) bis zur Realfahrt eingesetzt. Dabei stellt die Realfahrt aktuell die wichtigste Methode für die Freigabe dar; Ursache hierfür ist vor allem die Validität bei bisher noch vertretbarem ökonomischem Aufwand. Das autonome Fahren stellt jedoch auch, neben dem ökonomischen Aufwand, für die bekannten Methoden eine systematische Herausforderung dar. Aktuell steht die Realfahrt für die Fahrt im öffentlichen Straßenverkehr mit Testfahrer. Die Aufgabe des Testfahrers ist es, entsprechend der Aufgabe des Fahrzeugnutzers das Fahrzeug in jeder Situation zu führen oder zu überwachen. Übertragen auf das autonome Fahren wäre der Einsatz des Testfahrers am Fahrerarbeitsplatz ein nichtreales Verhalten eines Nutzers, denn dieser muss eben nicht mehr das Fahrzeug und das Umfeld überwachen und eingreifen. Außerdem könnte das Fahrzeug auch ohne Insassen am Straßenverkehr teilnehmen (abhängig vom Use-Case), sodass ein Testfahrer eine nichtreale Komponente im Fahrzeug darstellen würde. Deshalb besteht die Gefahr, dass durch den Einsatz eines Testfahrers die anderen Verkehrsteilnehmer beeinflusst werden und ein verändertes Verhalten zeigen. Weitere Gedanken zu diesem Thema sind in Kap. 7 zu finden.

Somit ist neben der Testfallgenerierung auch die aktuelle Testdurchführung nicht direkt auf das autonome Fahren übertragbar.

\subsubsection{Millionen Kilometer auf öffentlichen Straßen bis zur Freigabe des vollautomatischen Fahrens}

Folgende theoretische Betrachtung wird zeigen, was es bedeutet, trotz aufgezeigter Unterschiede dennoch das aktuelle Testkonzept beizubehalten. Angenommen, eine Raffung der 
Testfälle für das autonome Fahren ist nicht möglich, da keine Methode, ähnlich der Fahrerlaubnisprüfung des Menschen, existiert. Dennoch ist das Ziel, eine Aussage zu treffen, ob das Risiko durch den Einsatz des autonomen Fahrzeugs erhöht wird oder nicht:

$$
V_{a k z}=\frac{R_{z u s}}{R_{v e r}}<1
$$

Hier sei noch einmal darauf hingewiesen, dass diese Bedingung in keiner Weise zwingend ist. Für die theoretische Betrachtung wird die Bedingung kleiner eins jedoch als worst case angenommen.

Die einzige den Autoren bekannte belastbare Metrik für das Aufstellen eines solchen Verhältnisses sind die Zahlen der nachträglichen Auswertung von Verkehrsunfällen. Für Deutschland sind dies beispielsweise die Zahlen des Statistischen Bundesamtes. Für 2012 gibt das Statistische Bundesamt [21] beispielsweise 3375 polizeilich erfasste Unfälle mit Getöteten in Deutschland an. Dabei wird mit der Zahl für Getötete gearbeitet, da dies den worst case für den zu erbringenden Nachweis darstellt. Bezogen auf eine Fahrleistung von 709 Milliarden Kilometern in Deutschland, bedeutet diese Zahl als Durchschnittswert, dass zwischen zwei Unfällen mit Getöteten 210 Millionen Kilometer liegen. Da die Zahlen lediglich einen Erwartungswert darstellen, existieren auch Strecken zwischen zwei Unfällen, die kürzer oder länger sind. Um diese Verteilung der Unfallereignisse darzustellen, wird die Poisson-Verteilung herangezogen:

$$
P_{\lambda}(k)=\frac{\lambda^{k}}{k !} e^{-\lambda}
$$

Dabei wird angenommen, dass das Auftreten eines Unfalls ein unabhängiger und nicht erschöpfender Zufallsprozess ist $P_{\lambda}(k)$. In der Gleichung entspricht $k$ der Anzahl an Unfallereignissen und $\lambda$ dem Erwartungswert, mit dem dieses Ereignis auftritt. Der Erwartungswert $\lambda$ ist definiert durch den Quotienten

$$
\lambda=\frac{s_{\text {test }}}{s_{\text {leist }}}
$$

wobei $s_{\text {test }}$ für die beobachteten Testkilometer steht und $s_{\text {leist }}$ für die Leistungsfähigkeit des Systems. Die Leistungsfähigkeit steht für die erwartete Anzahl von Kilometern zwischen zwei Unfällen. Die Wahrscheinlichkeitsverteilungen für $k=\left[\begin{array}{llll}1 & 2 & 3 & 4\end{array}\right]$ und $\lambda=\left[\begin{array}{ll}1 & 2\end{array}\right]$ sind in Abb. 21.7 als Beispiel abgebildet.

Aus der Abbildung ist die Problematik für den Nachweis des Risikos gut zu erkennen: Angenommen, die blaue Verteilung steht für ein autonomes Fahrzeug und die rote Verteilung für ein Driver-Only-Fahrzeug. Beide Fahrzeuge werden die gleiche Anzahl von Testkilometern bewegt $s_{\text {test }}=a_{s} \cdot \bar{s}$, mit dem Streckenfaktor $a_{s}=2$ und dem durchschnittlichen Abstand $\bar{s}$ zwischen zwei Unfällen mit Getöteten. Die Leistungsfähigkeit $s_{\text {leist }}=a_{\text {leist }} \cdot \bar{s}$ 
Abb. 21.7 Poisson-Wahrscheinlichkeitsverteilung für die Anzahl von Unfällen mit unterschiedlichen Erwartungswerten

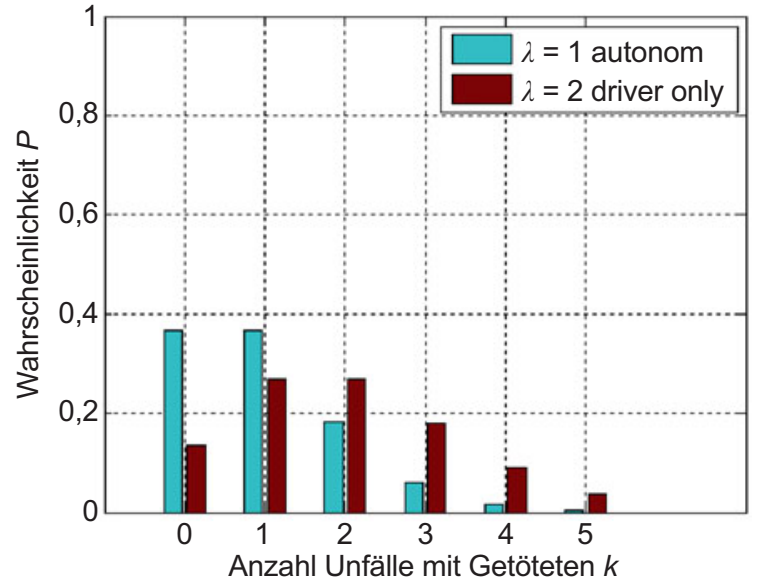

des autonomen Fahrzeugs ist um den Leistungsfaktor $a_{\text {leist }}=2$ größer als die des DriverOnly-Fahrzeugs. Daraus folgt für das autonome Fahrzeug der Erwartungswert $\lambda=1$ und für das Driver-Only-Fahrzeug $\lambda=2$.

Obwohl das autonome Fahrzeug gemäß der vorherigen Annahme eine doppelte Leistungsfähigkeit wie das Driver-Only-Fahrzeug auszeichnet, geriet das autonome Fahrzeug während des Tests in einen Unfall mit Getöteten (Wahrscheinlichkeit $P_{1}(1)=1 \cdot e^{-1} \approx 0,37$ ), das Driver-Only-Fahrzeug jedoch nicht (Wahrscheinlichkeit $P_{2}(0)=1 \cdot e^{-2} \approx 0,14$ ). Eine Aussage, dass das autonome Fahrzeug unsicherer sei als das Driver-Only-Fahrzeug, ist dementsprechend infrage zu stellen. Dieses Beispiel zeigt, dass auf jeden Fall ein Streckenfaktor $a_{s}$ größer zwei notwendig ist, um mit einer ausreichend hohen Signifikanz eine Aussage über die Leistungsfähigkeit des autonomen Fahrens treffen zu können.

Aus wissenschaftlicher Sicht ist beispielsweise von einer fünfprozentigen Irrtumswahrscheinlichkeit auszugehen und somit auch das gleiche Signifikanzniveau $e=5 \%$ heranzuziehen. Der Streckenfaktor $a_{s}$ ist in Abhängigkeit der Anzahl an Unfällen entsprechend groß zu wählen, sodass die Wahrscheinlichkeit für ein Fahrzeug mit geringerer Leistungsfähigkeit kleiner fünf Prozent wird, diese geringe Anzahl von Unfällen zu erreichen. Abb. 21.8 zeigt das Ergebnis dieser Überlegung und der numerischen Berechnung der Werte.

Der Datenpunkt bei null Unfällen mit Getöteten bedeutet, dass mit einem Streckenfaktor von $a_{s} \approx 3$ die Wahrscheinlichkeit kleiner fünf Prozent ist, dass ein schlechteres Fahrzeug als die Vergleichsgruppe in keinen Unfall mit Getöteten verwickelt ist.

Unglücklicherweise ist die Erfolgswahrscheinlichkeit für diesen Test genauso gering. Denn ist das Testfahrzeug genauso gut wie die Vergleichsgruppe, also gilt Leistungsfaktor $a_{\text {leist }}=1$, dann folgt daraus eine Erfolgswahrscheinlichkeit für diesen Nachweis von ebenfalls nur fünf Prozent. Damit der Test gelingt, ist eine größere Erfolgswahrscheinlichkeit erstrebenswert. Als Beispiel wird nun eine Erfolgswahrscheinlichkeit von 50 Prozent gefordert, mit der ein Test zeigen soll, dass das Testfahrzeug nicht schlechter als die Ver- 
Abb. 21.8 Streckenfaktor bei Signifikanzniveau fünf Prozent

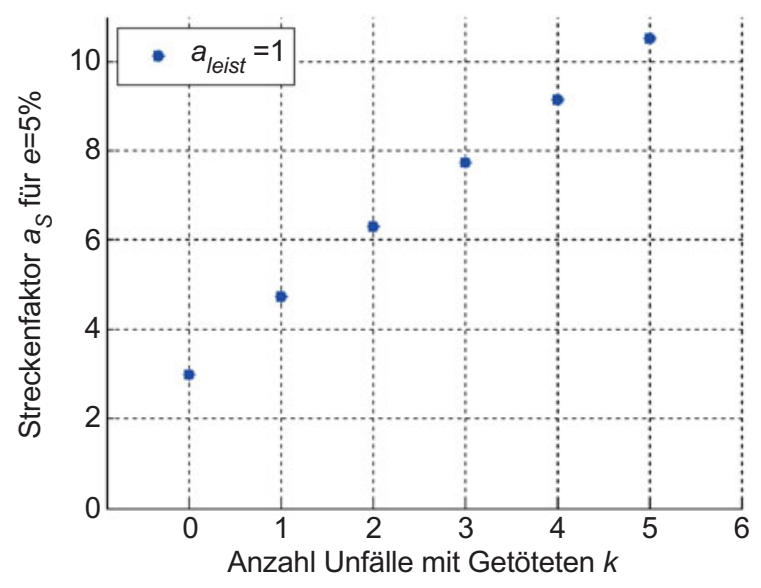

Abb. 21.9 Streckenfaktor über Leistungsfaktor bei einem Signifikanzniveau von fünf Prozent und einer Erfolgswahrscheinlichkeit des Tests von 50 Prozent

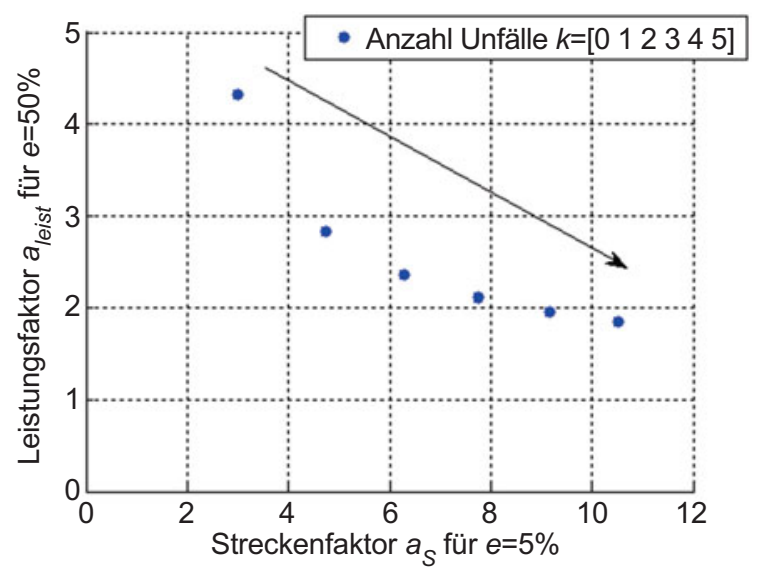

gleichsgruppe ist. Dazu muss das Testfahrzeug leistungsstärker sein als die Testgruppe. Abb. 21.9 zeigt das Ergebnis dieser Überlegung.

Der erste Punkt steht für folgende Aussage: Wenn das Testfahrzeug ca. 4,3-mal besser als die Vergleichsgruppe ist, dann gelingt der Test mit 50 Prozent Wahrscheinlichkeit, dass das Testfahrzeug mit einer Irrtumswahrscheinlichkeit von fünf Prozent besser als die Vergleichsgruppe ist.

Was dieses Ergebnis nun für die Testfahrt mit dem autonomen Fahrzeug bedeutet, wird an der Strecke zwischen zwei Unfällen mit Getöteten von 210 Millionen Kilometern demonstriert. Der letzte Punkt in Abb. 21.9 enthält die Aussage: Wenn das autonome Fahrzeug ca. zweimal so gut ist $\left(a_{\text {leist }} \approx 2\right)$ wie das Vergleichsystem (aktuelle Fahrzeuge), dann muss eine Teststrecke von mindestens 2,1 Milliarden Kilometern zurückgelegt werden $\left(s_{\text {test }}=a_{s} \cdot 210\right.$ Mio km). In diesem Fall wurde der Nachweis mit 50-prozentiger Wahrscheinlichkeit erbracht, allerdings wurden auch mit der gleichen Wahrscheinlichkeit fünf Unfälle erfahren. 
Aus dieser Überlegung folgt ironischerweise, dass je leichter die Fahrzeugführung ist, desto mehr Testkilometer absolviert werden müssten, da der Vergleichswert entsprechend größer ist. Für den Autobahnpiloten folgt aus den aktuellen Zahlen des Statistischen Bundesamtes ein Vergleichswert von 662 Millionen Kilometern zwischen zwei Unfällen mit Getöteten. Dementsprechend müssten 6,62 Milliarden Testkilometer auf der Autobahn absolviert werden, um den dargestellten Bedingungen zu entsprechen.

Dieser theoretische Exkurs in die Statistik zeigt, dass die Freigabe zur Herausforderung, wenn nicht sogar zur Falle für das autonome Fahren werden kann. Dabei wurden mehrere Faktoren für die Ermittlung der Testkilometer noch nicht adressiert; beispielsweise würde eine Variation des Systems zu einem erneuten Fahren der Testkilometer führen, oder der Test mit und ohne Insassen könnte mit einem Faktor zwei in die Berechnung eingehen. Wie sich die unterschiedlichen, hier nicht betrachteten Parameter wie Einsatzbereich, Unfallfolgentyp, Unfallverursachung und Vergleichsfahrzeuge auf die ermittelten notwendigen Kilometer auswirken, wird in Winner [22] detailliert hergeleitet.

Bei diesen Überlegungen handelt es sich um eine theoretische Betrachtung mit frei getroffenen Annahmen. Dennoch eignet sich dieser Ansatz, um die Problematik und die Herausforderungen aufzuzeigen sowie die nun folgenden Ansätze zu motivieren.

\subsection{Ansätze für das Lösen der Freigabeherausforderung}

Wie gezeigt wurde, bildet das autonome Fahren ein neues Testobjekt, das aufgrund seiner Eigenschaften den Einsatz der klassischen Testkonzepte infrage stellt. Das Überwinden der beschriebenen Freigabeherausforderung bedarf neuer Ansätze: Dementsprechend wird im nächsten Unterabschnitt diskutiert, warum aus Sicht der Freigabe das Wiederverwenden von freigegebenen Funktionen und somit ein evolutionärer Ansatz notwendig erscheint. Daran anschließend werden existierende Ansätze diskutiert, die eine Beschleunigung der Freigabe ermöglichen könnten.

\subsubsection{Wiederverwenden freigegebener Funktionen}

Die erste und einfachste Möglichkeit, eine Freigabe für ein neues System zu erhalten, ist das Wiederverwenden von bereits freigegebenen Funktionen. Wird ein System in gleicher Weise wie zuvor eingesetzt, so kann die bereits erteilte Freigabe übernommen werden. Ein erweiterter Funktionsumfang ist jedoch neu abzusichern; je kleiner dabei der neue Bereich ist, desto geringer wird der Aufwand.

Dieser Argumentation folgend scheint eine Evolution über alle Dimensionen als möglicher Ansatz, um die Freigabeherausforderung zu beherrschen. Mit den Dimensionen ist hier beispielsweise die Geschwindigkeit, der Einsatzbereich, aber auch der Automationsgrad gemeint. Bei der Wahl der Evolutionsschritte können zwei Ansichten unterschieden werden: Aus Sicht eines Funktionsentwicklers ist die Autobahn während 
eines Stauszenarios aufgrund der reduzierten Geschwindigkeit und der begrenzt zugänglichen Szenerie ein geeignetes Einstiegszenario. Aus Sicht der zuvor angestellten statistischen Überlegungen wäre ein Einstiegszenario sinnvoll, bei der der Mensch als Vergleichsgruppe möglichst schlecht abschneidet, also möglichst viele Fehler macht. Möglichst viele Fehler bedeutet wenig Strecke, sodass der Nachweis der Leistungsfähigkeit einfacher wird.

Der revolutionäre Schritt - ein autonomes Fahrzeug ohne evolutionäre Zwischenschritte abzusichern - widerspricht diesem Ansatz und scheint unwahrscheinlich.

\subsubsection{Beschleunigung der Freigabe}

Trotz des evolutionären Ansatzes sind dennoch neue Funktionen abzusichern. Um dies zu beschleunigen, sind prinzipiell zwei Stellschrauben vorhanden: Erstens kann das Was und zweitens das Wie verändert werden. Welche Testfälle sind notwendigerweise zu überprüfen und womit werden diese Tests durchgeführt? Schuldt et al. [10] nennen dies Testfallgenerierung und Testdurchführung.

\subsubsection{Testfallgenerierung}

Die Testfallgenerierung definiert die durchzuführenden Tests: Nach Schuldt et al. [10] folgt aus der Vielzahl von Einflussfaktoren im Einsatzbereich sowie deren Wertebereichen eine unüberschaubare Anzahl von Testfällen. Wie bereits beschrieben, stützen sich Systeme, die aktuell im Einsatz sind, auf die Leistungsfähigkeit des Menschen und dessen Möglichkeit, das Fahrzeug zu kontrollieren. Daraus folgt eine starke Raffung der theoretisch notwendigen Testfälle. Somit existiert eine Metrik, die eine Aussage über die Sicherheit ermöglicht, ohne sämtliche Situationen zu testen. Diese Raffung entfällt für das autonome Fahrzeug, sodass neue Wege gesucht werden, die Anzahl von Testfällen für das autonome Fahrzeug zu reduzieren. Dabei sind während der Testfallgenerierung die Anforderungen an ein Testkonzept aus Abschn. 21.3 zu beachten. Besonders die Repräsentativität ist gefährdet, wenn Testfälle gestrichen werden.

Die Ansätze von Glauner [23] und Eckstein [24] beschreiben dafür das Identifizieren von relevanten bzw. kritischen Situationen im öffentlichen Straßenverkehr: Basierend auf zuvor definierten Ereignisklassen werden während der Testfahrten oder groß angelegter Feldstudien potenziell kritische Situationen identifiziert. Diese kritischen Situationen fließen in die Testfallgenerierung ein, sodass Situationen geringer Kritikalität gestrichen werden können. Dieser Raffung liegt die Annahme zugrunde, dass Situationen, die weniger kritisch sind, durch kritische Situationen abgedeckt werden. Dabei bleibt aktuell als ungelöste Aufgabe die Suche nach einem validen Risikomaß, das im ersten Schritt eine Bewertung und im zweiten Schritt die Auswahl von kritischen Situationen ermöglicht.

Ein anderes Vorgehen zur Raffung von Testfällen bieten Schuldt et al. [10]: Vorgeschlagen wird eine generische Testfallgenerierung, bei der Verfahren des Black-Box-Testings und der Kombinatorik eingesetzt werden, um die Einflussfaktoren auf die vom System ausgehende 
Sicherheit möglichst ausreichend abzudecken und gleichzeitig redundanzarm und effizient zu sein. Dieser Ansatz basiert auf statistischen Betrachtungen ohne Wissen und Erfahrung über das Testobjekt, hat aber dennoch das Potenzial, die notwendigen Testfälle zu reduzieren.

Der Ansatz beschrieben von Tatar und Mauss [25] ist ebenfalls für Black-Box-Testing geeignet: Zur Generierung von Testfällen wird eine Optimierung eingesetzt. Dabei werden die Eingangsgrößen einer X-in-the-Loop (XiL)-Simulation so variiert, dass die zu definierende Bewertungsfunktion des Tests optimiert wird. Trotz der Herausforderung der validen XiL-Simulation und der benötigten Bewertungsfunktion liefert dieser Ansatz die Möglichkeit, die Testfälle auf die als relevant bewerteten zu fokussieren.

Ein vierter theoretischer Ansatz ist der Einsatz und der Test eines Sicherheitskonzepts mithilfe von formalen Methoden [26]. Ähnlich wie für den Menschen als Überwacher und Teil des Sicherheitskonzepts von aktuellen Fahrzeugen angenommen, könnte ein nachgewiesen sicheres Sicherheitskonzept den Test der Gesamtfunktionalität des Fahrzeugs in der vollständigen Repräsentativität überflüssig machen. Somit wäre eine Raffung der Testfälle möglich.

\subsubsection{Testdurchführung/Testwerkzeug}

Neben der Möglichkeit, während der Testfallgenerierung die Testfälle zu raffen, besitzt die Testdurchführung ebenfalls Potenzial, die Freigabe zu beschleunigen. Wird jedoch von der Realfahrt abgewichen und ein anderes Testwerkzeug für die Testdurchführung gewählt, geht damit immer eine Vereinfachung einher. Dies wird anhand von Abb. 21.10 genauer beschrieben.

Abb. 21.10 unterteilt die Testwerkzeuge in neun Klassen, die sich danach unterscheiden, wie das Fahrzeug bzw. das Umfeld dargestellt werden. Der Insasse wird bei dieser Darstellung dem Fahrzeug zugeordnet, da er sich im Fahrzeug befindet und nicht aktiv in die autonome Fahrt eingreift.

Die Realfahrt bildet sowohl Umfeld als auch Fahrzeug real ab. Dementsprechend besteht während dieser Tests die Gefahr von realen Unfällen und deren Auswirkungen. Das Umfeld ist nicht kontrolliert, sodass Testsituationen basierend auf dem Zufall der Realität entstehen; dementsprechend ist die Reproduzierbarkeit für komplexe Situationen mit anderen Verkehrsteilnehmern nicht gegeben. Dieses Testwerkzeug kann frühestens mit ersten straßentauglichen Prototypen eingesetzt werden und erfolgt somit am Ende des Entwicklungsprozesses.

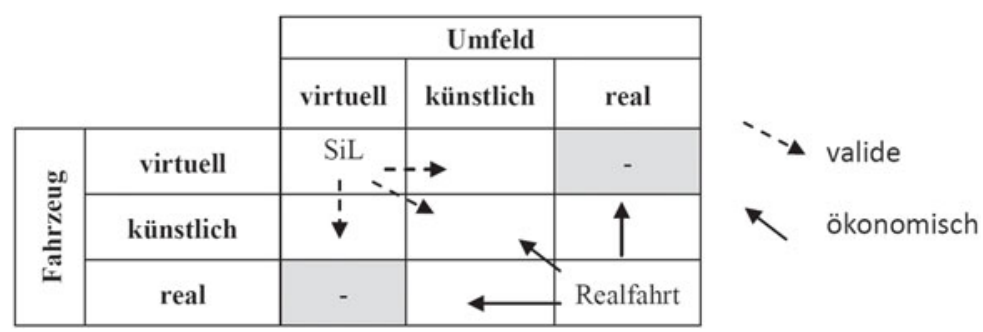

Abb. 21.10 Einteilung von Testwerkzeugen für den Test von autonomen Fahrzeugen 
Eine Alternative ist das Testen von realen Fahrzeugen in einem künstlichen Umfeld: Dies entspricht der Fahrt auf einem Testfeld, denn dort sind zum einen Situationen künstlich hergestellt und zum anderen besitzen die „Verkehrsteilnehmer“ das Bewusstsein, sich in einem Test zu befinden. Zugunsten der Sicherheit, Variierbarkeit, Beobachtbarkeit sowie der Reproduzierbarkeit wird die Realität vereinfacht. Aus ökonomischen Gesichtspunkten werden zwar Testfälle gezielt getestet und müssen nicht wie in Realfahrten per Zufall erfahren werden, jedoch bedarf der Aufbau des Testfelds zusätzliche zeitliche wie finanzielle Mittel.

Des Weiteren könnte sich ein künstliches Fahrzeug in einem realen Umfeld bewegen; künstlich bezieht sich in diesem Fall beispielsweise auf die Ausstattung des autonomen Fahrzeugs mit einem Überwacher, der die Möglichkeit hat, in die Fahraufgabe einzugreifen. Dies kann zum einen ein Testfahrer mit Lenkrad und Pedalerie sein oder aber ein technisches System, das aufgrund von leistungsfähigerer (Zusatz-)Sensorik dem Seriensystem überlegen ist. Werden Komponenten künstlich dargestellt, leidet darunter die Realitätsnähe, jedoch wird an Sicherheit, Reproduzierbarkeit und Beobachtbarkeit gewonnen.

Neben der Möglichkeit, Umfeld und Fahrzeug künstlich zu gestalten, existieren Werkzeuge, die sich einer virtuellen Repräsentation in Form von Computersimulationen bedienen. Dabei sind die zwei Felder, die real und virtuell kombinieren, grau hinterlegt, da diese streng genommen nicht existieren, denn Sensoren und Steller haben genau die Aufgabe der Wandlung zwischen virtuellen und realen Signalen. Ein realer Radarsensor kann kein virtuelles Umfeld sensieren, und ein virtueller Wechselrichter kann keine reale Spannung erzeugen.

Was jedoch möglich ist, sind Kombinationen aus künstlichem und virtuellem Umfeld bzw. Fahrzeug: Als Beispiel dafür existieren unterschiedliche Konzepte von Vehicle-in-theLoop (ViL). Um den Kreis aus Aktionen und Reaktionen von Umfeld und Fahrzeug zu schließen, werden reale Komponenten in der Simulation in Form von Modellen abgebildet. Dabei werden entweder die angesprochenen Sensoren bzw. Steller stimuliert, also künstlich angeregt; Beispiele hierfür sind simulationsbasierte Videos als Stimulanz für Kamerasysteme oder Rollenprüfstände als Stimulanz für Antriebssteller. Oder die Testwerkzeuge simulieren direkt die Leistungssignale wie z.B. die elektromagnetische Welle und versuchen, reale Effekte von Sensoren und Stellern in der Simulation mithilfe von Modellen darzustellen. Für weitere Informationen dazu s. Bock [27] oder Hendricks [28]. Der beschriebene Einsatz von Modellen stellt die Aussagekraft dieser Testwerkzeuge infrage. Um valide Aussagen mithilfe solcher Modelle zu erhalten, ist der Nachweis zu erbringen, dass diese Modelle keine unzulässigen Vereinfachungen beinhalten; „unzulässig“ ist hier im Kontext der Funktion zu sehen und bedeutet, dass Abweichungen von der Realität nur unterhalb der Toleranzen der Funktion zulässig sind. Wenn jedoch diese Validität nachgewiesen wurde, erlaubt das Testwerkzeug eine größere Sicherheit bei der Testdurchführung, da sich Teile des Umfelds und das Fahrzeug nur noch in der virtuellen Welt begegnen. Aufgrund der virtuellen Komponenten sind diese Testwerkzeuge von einer größeren Variierbarkeit, Beobachtbarkeit und Reproduzierbarkeit gekennzeichnet. Aus ökonomischer Sicht besitzt dieses Testwerkzeug den Vorteil, das virtuelle Umfeld einfach zu variieren 
oder aber das Fahrzeug in unterschiedlichsten Varianten darzustellen. Von ökonomischem Nachteil könnte sich die Validierung der Modelle erweisen (vgl. Herausforderung bei SiL). Ein Vorteil dieses Testwerkzeugs ist die Möglichkeit, basierend auf dem simulierten Fahrzeug, bereits frühzeitig in der Entwicklung Tests durchzuführen.

Die letzte Stufe der Abstraktion repräsentiert die Kombination eines virtuellen Fahrzeugs und des virtuellen Umfelds: Das mit Software-in-the-Loop (SiL) bezeichnete Testwerkzeug stellt dabei den geschlossenen Regelkreis durch die Modellierung relevanter Komponenten in der Simulation dar. Anders als bei den Testwerkzeugen zuvor, ist die gesamte Testwelt virtuell. Die Tests sind sicher, variierbar, beobachtbar und reproduzierbar; außerdem besteht die Möglichkeit, dieses Werkzeug bereits frühzeitig in der Entwicklung einzusetzen. Den ökonomischen Vorteil bildet die Hardware-Unabhängigkeit, denn somit ist keine Verknüpfung an die Echtzeit mehr vorhanden. Die Ausführung der Tests wird rein durch Rechenleistung begrenzt; Simulationen können Tag und Nacht sowie massiv parallel erfolgen. Demgegenüber steht die Realitätsnähe der virtuellen Testwelt und somit jedes einzelnen Modells: Nur wenn die Validität der eingesetzten Modelle nachgewiesen wurde, besitzen virtuelle Tests die Aussagekraft für eine Freigabe. Dementsprechend muss für die ökonomische Betrachtung von simulationsbasierten Verfahren vor allem die Validierung der Modelle betrachtet werden.

Die gleiche Herausforderung existiert für den Einsatz von formalen Methoden. Mitsch schreibt diesbezüglich: "We do ... prove that collisions can never occur (as long as the robot system fits to the model)." [26] Das bedeutet, auch für formale Methoden bedingt der Realitätsgrad der eingesetzten Modelle die Aussagekraft der Ergebnisse. Eine besondere Herausforderung und somit im Fokus der Forschung steht beispielsweise die Formalisierung der Unsicherheiten von Sensoren oder der Eigenschaft von weiteren Verkehrsteilnehmern.

Die Diskussion der Testwerkzeuge zeigt das Potenzial, die Freigabe zu beschleunigen: Mithilfe von künstlich erzeugtem Umfeld und Fahrzeug können Testfälle gezielt aufgebaut und angefahren werden. Zusätzlich ermöglicht der virtuelle Ansatz, die Tests abhängig von der eingesetzten Rechenleistung zu beschleunigen und zu parallelisieren.

Die Diskussion zeigt aber auch, dass die Validität und somit die Aussagekraft der Tests mit Einführung von künstlichen und virtuellen Komponenten zur Herausforderung wird.

\subsection{Fazit}

Das autonome Fahren unterscheidet sich besonders durch den Wegfall des menschlichen Überwachers von assistierten oder teilautomatisierten Systemen und dessen Fähigkeiten zur Korrektur dieser Systeme. Die Metrik, bestehend aus Realfahrt und Fahrerlaubnistest, die eine Aussage über die Sicherheit aktuell in Serien befindlicher Automatisierungsgrade ermöglicht, ist für das autonome Fahren nicht mehr gültig. Der daraus folgende Wegfall der Raffung von Testfällen führt dazu, dass aktuelle Testkonzepte nicht geeignet sind, um das neue System des autonomen Fahrens abzusichern. Das Festhalten an aktuellen Testkonzepten würde zu einem ökonomisch nicht vertretbaren Aufwand führen und zur Frei- 
gabefalle für das autonome Fahren werden. Die Autoren sehen jedoch drei Ansätze, diese Freigabefalle zu umgehen:

Erstens erscheint der evolutionäre Ansatz oder alternativ die Transformation (s. Kap. 10) als notwendig, denn nur die schrittweise Einführung entlang der unterschiedlichen Dimensionen Geschwindigkeit, Szenerie und Automationsgrad ermöglicht das Übernehmen von abgesicherten Komponenten und reduziert den Aufgabenumfang der folgenden Freigaben. Zweitens sind die notwendigen Testfälle, basierend auf Felderfahrung und statistischen Verfahren zu raffen. Die Herausforderung ist dabei die Metrik, die eine Aussage über die Sicherheit des Systems in Abhängigkeit der absolvierten Testfälle ermöglicht. Drittens sind alternative Testwerkzeuge neben der Realfahrt einzusetzen. Dabei wird erwartet, dass auf Realfahrten nicht vollständig verzichtet werden kann, denn die Verlagerung von Testfällen in ViL, SiL bzw. in Verfahren des formalen Beweises von Sicherheit bedarf den Nachweis der Validität.

Abschließend ist zu sagen, dass die vorgestellten Herausforderungen nicht ausschließlich intern durch die Automobilindustrie gelöst werden sollten. Auch wenn Testkonzepte für das autonome Fahren optimiert werden, wird es die 100-prozentige Sicherheit nicht geben. Die „Vision Zero“ bleibt besonders im Mischbetrieb mit zusätzlichen Verkehrsteilnehmern zunächst eine Vision. Spätestens mit dem ersten Unfall, den ein autonomes Fahrzeug verursacht, wird die zuvor erteilte Freigabe auf den Prüfstand gestellt. Dementsprechend sollte die Grundlage für die Freigabe durch die Beteiligten öffentlich diskutiert und transparent gestaltet werden.

\section{Literatur}

1. Felkai, R., Beiderwieden, A.: Schaffen allgemeiner Voraussetzungen der Projektabwicklung. In: Projektmanagement für technische Projekte, pp. 7-49. Springer Fachmedien Wiesbaden (2013)

2. Das Europäische Parlament und der Rat der Europäischen Union: Richtlinie 2007/46/EG (2007)

3. Reuter, A.: Produkthaftung in Deutschland. In: Werdich, M. (ed.) FMEA - Einführung und Moderation, pp. 121-137. Vieweg+Teubner Verlag (2012)

4. Gasser, T.M., Arzt, C., Ayoubi, M., Bartels, A., Bürkle, L., Eier, J., Flemisch, F., Häcker, D., Hesse, T., Huber, W., Lotz, C., Maurer, M., Ruth-Schumacher, S., Schwarz, J., Vogt, W.: Rechtsfolgen zunehmender Fahrzeugautomatisierung. Gemeinsamer Schlussbericht der Projektgruppe. Berichte der Bundesanstalt für Strassenwesen - Fahrzeugtechnik (F), vol. 83. Wirtschaftsverl. NW Verl. für neue Wissenschaft, Bremerhaven (2012)

5. Ward, R.B.: Revisiting Heinrich's law. In: Chemeca. Quality of life through chemical engineering (2012)

6. Hydén, C.: The development of a method for traffic safety evaluation: The Swedish traffic conflicts technique, Lund Institute of Technology. Department of Traffic Planning and Engineering (1987)

7. Donner, E., Winkle, T., Walz, R., Schwarz, J.: RESPONSE 3 - Code of Practice für die Entwicklung, Validierung und Markteinführung von Fahrerassistenzsystemen. In: VDA Technischer Kongress 2007, pp. 231-241, Sindelfingen (2007) 
8. Spanfelner, B., Richter, D., Ebel, S., Wilhelm, U., Branz, W., Patz, C.: Herausforderungen in der Anwendung der ISO26262 für Fahrerassistenzsysteme. Challenges in applying the ISO 26262 for driver assistance systems. In: Lehrstuhl für Fahrzeugtechnik, TU München (ed.) 5. Tagung Fahrerassistenz, München (2012)

9. Weitzel, A., Winner, H., Peng, C., Geyer, S., Lotz, F., Sefati, M.: Absicherungsstrategien für Fahrerassistenzsysteme mit Umfeldwahrnehmung, Berichte der Bundesanstalt für Straßenwesen - Fahrzeugtechnik (F), vol. 98. Wirtschaftsverl. NW Verl. für neue Wissenschaft, Bremerhaven (2014)

10. Schuldt, F., Saust, F., Lichte, B., Maurer, M., Scholz, S.: Effiziente systematische Testgenerierung für Fahrerassistenzsysteme in virtuellen Umgebungen. In: AAET 2013

11. Horstmann, M.: Verflechtung von Test und Entwurf für eine verlässliche Entwicklung eingebetteter Systeme im Automobilbereich, TU Braunschweig (2005)

12. Daimler AG: Mercedes-Benz präsentiert in Genf Limousine und Coupé der neuen E-Klasse (2009)

13. Fach, M., Baumann, F., Breuer, J., May, A.: Bewertung der Beherrschbarkeit von Aktiven Sicherheits- und Fahrerassistenzsystemen an den Funktionsgrenzen. In: 26. VDI/VW-Gemeinschaftstagung Fahrerassistenz und Integrierte Sicherheit, 6./7. Oktober 2010 in Wolfsburg (2010)

14. Burgdorf, F.: Eine kunden- und lebenszyklusorientierte Produktfamilienabsicherung für die Automobilindustrie, KIT Scientific Publishing; Karlsruher Institut für Technologie (2010)

15. Wirtschaftskommission der Vereinten Nationen für Europa (UN/ECE): Regelung Nr. 13-H Einheitliche Bedingungen für die Genehmigung von Personenkraftwagen hinsichtlich der Bremsen (2010)

16. Baake, U., Wüst, K., Maurer, M., Lutz, A.: Testing and simulation-based validation of ESP systems for vans. ATZ Worldw 116(2), 30-35 (2014). doi: 10.1007/s38311-014-0021-6

17. Rasmussen, J.: Skills, Rules, and Knowledge; Signals, Signs, and Symbols, and Other Distinctions in Human Performance Models. IEEE Transactions On Systems, Man, and Cybernetics SMC-13(3), 257-266 (1983)

18. Donges, E.: Fahrerverhaltensmodelle. In: Winner, Hakuli, Wolf (eds.) Handbuch Fahrerassistenzsysteme, pp. 15-23 (2011)

19. Ständer, T.: Eine modellbasierte Methode zur Objektivierung der Risikoanalyse nach ISO 26262 , TU Braunschweig (2011)

20. Bahr, M., Sturzbecher, D.: Bewertungsgrundlagen zur Beurteilung der Fahrbefähigung bei der praktischen Fahrerlaubnisprüfung. In: Winner, H., Bruder, R. (eds.) 6. Darmstädter Kolloquium Mensch+Fahrzeug: Maßstäbe des sicheren Fahrens. Ergonomia (2013)

21. Statistisches Bundesamt (Destatis): Verkehrsunfälle - Fachserie 8 Reihe 7 (2012)

22. Winner, H.: Quo vadis, FAS? In: Winner, H., Hakuli, S., Lotz, F., Singer, C. (eds.) Handbuch Fahrerassistenzsysteme, 3rd edn. Vieweg-Teubner-Verlag (2015)

23. Glauner, P., Blumenstock, A., Haueis, M.: Effiziente Felderprobung von Fahrerassistenzsystemen. In: UNI DAS e.V (ed.) 8. Workshop Fahrerassistenzsysteme, pp. 5-14, Walting (2012)

24. Eckstein, L., Zlocki, A.: Safety Potential of ADAS - Combined Methods for an Effective Evaluation. ESV (2013)

25. Tatar, M., Mauss, J.: Systematic Test and Validation of Complex Embedded Systems. ERTS2014, Toulouse, 5-7 (2014)

26. Mitsch, S., Ghorbal, K., Platzer, A.: On Provably Safe Obstacle Avoidance for Autonomous Robotic Ground Vehicles. Robotics Science and Systems (RSS), 2013. Accessed 27 June 2014

27. Bock, T.: Bewertung von Fahrerassistenzsystemen mittels der Vehicle in the Loop-Simulation. In: Winner, H., Hakuli, S., Wolf, G. (eds.) Handbuch Fahrerassistenzsysteme, pp. 76-83. Vieweg+Teubner Verlag (2012)

28. Hendriks, F., Tideman, M., Pelders, R., Bours, R., Liu, X. (eds.): Development tools for active safety systems: Prescan and VeHIL. Vehicular Electronics and Safety (ICVES), 2010 IEEE International Conference on (2010) 